\title{
The role of emergency department triage early warning score (TREWS) and modified early warning score (MEWS) to predict in-hospital mortality in COVID-19 patients
}

\author{
Huseyin Aygun ${ }^{1}\left(\mathbb{D} \cdot\right.$ Suna Eraybar ${ }^{1,2}(\mathbb{D}$ \\ Received: 1 June 2021 / Accepted: 19 June 2021 / Published online: 28 June 2021 \\ (c) Royal Academy of Medicine in Ireland 2021
}

\begin{abstract}
Background It is necessary to identify critical patients requiring hospitalization early due to the rapid increase in the number of COVID-19 cases.

Aim This study aims to evaluate the effectiveness of scoring systems such as emergency department triage early warning score (TREWS) and modified early warning score (MEWS) in predicting mortality in COVID-19 patients.

Methods In this retrospective cohort study, PCR positive patients evaluated for COVID-19 and decided to be hospitalized were evaluated. During the first evaluation, MEWS and TREWS scores of the patients were calculated. Intensive care needs as well as 24-h and 28-day mortality rates were evaluated.

Results A total of 339 patients were included in the study. While $30(8.8 \%)$ patients were hospitalized in the intensive care unit, 4 (1.2\%) died in the emergency. The number of patients who died within 28 days was found to be 57 (16.8\%). In 24-h mortality, the median MEWS value was found to be 7 (IQR 25-75) while the TREWS value was 11.5 (IQR 25-75). In the ROC analysis made for the diagnostic value of 28-day mortality of MEWS and TREWS scores, the area under the curve (AUC) for the MEWS score was found to be 0.833 (95\% CI $0.777-0.888, p<0.001)$ while it was identified as $0.823(95 \%$ CI $0.764-0.882, p<0.001)$ for the TREWS.

Conclusion MEWS and TREWS calculated at emergency services are effective in predicting 28-day mortality in patients requiring hospitalization due to COVID-19.
\end{abstract}

Keywords COVID- $19 \cdot$ Early warning scores $\cdot$ Emergency $\cdot$ MEWS $\cdot$ Mortality $\cdot$ TREWS

\section{Introduction}

To determine the severity of the disease of patients admitted to emergency departments, different triage systems are used worldwide. These systems may guide emergency professionals in terms of the seriousness of diagnostic processes and have an important role in the functioning of emergency

Suna Eraybar

sunaeraybar@gmail.com

Huseyin Aygun

drhaygun@gmail.com

1 Emergency Department, University of Health Sciences Turkey, Bursa Yuksek Ihtisas Training and Research Hospital, Bursa, Turkey

2 Emergency Department, Yuksek Ihtisas Education and Research Hospital, Mimar Sinan Mah. , Emniyet Cad. Polis Okulu Karşısı Yildirim, Bursa, Turkey services in effective and rapid treatment planning. Today, many scoring systems are being used to determine hospitalization needs of emergency patients and to predict in-hospital mortality.

Modified early warning score (MEWS), rapid emergency medicine score (REMS), and rapid acute physiology score (RAPS) are among the most commonly used scoring systems in emergencies [1,2]. Subble et al. developed MEWS in 2001 through the modification of the early warning score [3]. Systolic blood pressure is evaluated by scoring the parameters of heart rate, respiratory rate, body temperature, and AVPU (A: alert, V: verbal, P: pain, $\mathrm{U}$ : unresponsive) between 0 and 3 points. No consensus has been reached regarding the results of the MEWSrelated studies. It is frequently used in critically ill patient identification and mortality prediction in intensive care and emergency services [4-6]. However, its prognostic 
effectiveness has been found to be unsuccessful in studies conducted with different patient groups [7, 8].

In studies on the efficiency assessment of MEWS, the cutoff value in predicting in-hospital mortality varies $[1$, $4,7]$. Additionally, in studies on nontraumatic emergency patients, the cutoff value to predict mortality has been identified to be varied between 2 and 5 . In another study conducted on intensive care patients, it has been found that when the MEWS is $\geq 6$, it can predict mortality [9-11]. This suggests that the performance and effective cutoff value of MEWS may differ in particular patient groups.

In studies on COVID-19 patients with PCR positive test results, the MEWS, which was evaluated during the first admission to the emergency department, has been found to be effective in determining the need for intensive care hospitalization [12, 13]. In addition, it has been evaluated as an important parameter in determining in-hospital mortality especially in COVID-19 patients over 65 [14].

In these days, as the coronavirus pandemic has been continuing, the scoring systems that are used in the identification of critically ill patients in emergency departments have gained more importance. Moreover, they have contributed more to the rapid and effective use of existing potential.

Considering the information obtained about COVID-19, it is now known that low $\mathrm{SPO} 2$ is an important cause of mortality [15]. Studies have found that scoring systems such as REMS, which includes the $\mathrm{SPO} 2$ parameter, are superior to MEWS in predicting mortality in COVID-19 patients evaluated in emergency services $[13,16]$. Additionally, independent of oxygen saturation, the TREWS, which includes the oxygen need and patient age among its parameters, may be effective in predicting severity in COVID-19 patients admitted to emergencies.

TREWS is another important scoring system developed by Lee et al. in 2020. It has been developed by evaluating the efficiency of the national early warning score that are used as an important prognostic indicator. It includes physiological parameters such as respiratory rate, oxygen saturation, need for oxygen support, body temperature, systolic blood pressure, heart rate, and consciousness. Peripheral oxygen saturation is excluded as it is insufficient in determining the severity in all groupings among the parameters, even at the lowest rates and final multivariable analyses are performed by including the age of patients in the assessment. Compared to MEWS, TREWS has been found to be more effective in predicting in-hospital mortality [17]. In the literature regarding TREWS, a newly developed scoring system, no evaluation related to COVID-19 has been encountered.

The aim of our study is to evaluate the prognostic significance of MEWS and TREWS which are calculated during the admission of hospitalized COVID-19 patients in predicting mortality.

\section{Material and methods}

Patients over the age of 18 who were evaluated for COVID-19 due to the positive PCR performed through a combined nasal and throat swab and who underwent thorax computed tomography (TCT) and then were hospitalized were retrospectively screened. In the planning stage of the study, necessary permissions were obtained from the ethics committee of our hospital (2011-KAEK-25 2020 / 05-16) and from the XX Ministry of Health, General Directorate of Health Services. Patients who were under the age of 18 , pregnant, and who had a negative PCR were excluded.

Selected patients' age, gender, chronic disease, symptoms, vital signs measured at the time of arrival, TCT findings, and hospitalization information were recorded through the patient files registered in the hospital automation system. PCR test results were recorded over the Public Health Management System, Case Tracking Module of the Republic of Turkey Ministry of Health.

TCT results of the patients were recorded as typical (peripheral, bilateral ground glass opacity (GGO) with or without consolidation or visible intralobular lines, multifocal GGO of rounded morphology or visible intralobular lines, reverse halo sign, or other findings of organizing pneumonia), intermediate, atypical, and negative in accordance with the Radiological Society of North America Expert Consensus Statement on Reporting Chest CT Findings Related to COVID-19 classification [15].

MEWS and TREWS were calculated as a result of the vital signs and evaluations of the patients at the first admission. MEWS, TREWS, and their components are summarized in Table 1. The relationship between MEWS and TREWS scores with in-hospital, 14-day, and 28-day mortality was evaluated. The effectiveness of scoring systems in predicting mortality was compared.

\section{Statistical analysis}

IBM SPSS Statistics for Windows, Version21.0 (IBM Corp. Armonk, NY: USA, released 2012) was used for statistical analysis. While descriptive statistics were expressed as mean \pm standard deviation (minimum-maximum), median and range, and/or interquartile range (IQR) for numerical variables, categorical variables were presented as the number of cases and (\%). Kolmogorov-Smirnov test was used for the normality distribution of the data. Whether the assumption of homogeneity of variances was met was investigated by Levene's test. The significance of the difference between the groups in terms of continuous numerical variables where parametric test statistics assumptions were met was examined with the Student's $t$ test, while the significance of the 
Table 1 MEWS and TREWS components

\begin{tabular}{|c|c|c|c|c|c|c|c|}
\hline Variable & 3 & 2 & 1 & 0 & 1 & 2 & 3 \\
\hline \multicolumn{8}{|c|}{ Systolic blood pressure (mmHg) } \\
\hline MEWS & $<70$ & $71-80$ & $81-100$ & 101-199 & & \multicolumn{2}{|l|}{$\geq 200$} \\
\hline TREWS & $\leq 90$ & $91-100$ & $101-110$ & $\geq 111$ & & & \\
\hline \multicolumn{8}{|c|}{ Heart rate (bmp) } \\
\hline MEWS & & $<40$ & $41-50$ & $51-100$ & $101-110$ & $111-129$ & $\geq 130$ \\
\hline TREWS & & & & $\leq 110$ & $111-120$ & $\geq 121$ & \\
\hline \multicolumn{8}{|c|}{ Respiratory rate (bmp) } \\
\hline MEWS & & $<9$ & & $9-14$ & $15-20$ & $21-29$ & $\geq 30$ \\
\hline TREWS & $\leq 8$ & $9-11$ & $12-17$ & $18-24$ & & $\geq 25$ & \\
\hline \multicolumn{8}{|c|}{ Body temperature $\left({ }^{\circ} \mathrm{C}\right)$} \\
\hline MEWS & & $<35$ & & $35-38.4$ & & $\geq 38.5$ & \\
\hline TREWS & $\leq 35$ & $35.1-36$ & & $\geq 36$ & & & \\
\hline \multicolumn{8}{|c|}{ AVPU score } \\
\hline MEWS & & & & A & $\mathrm{V}$ & $\mathrm{P}$ & $\mathrm{U}$ \\
\hline TREWS & & & & A & & & $\mathrm{V}, \mathrm{P}, \mathrm{U}$ \\
\hline \multicolumn{8}{|c|}{ Oxygen supply } \\
\hline MEWS & - & - & - & - & - & - & - \\
\hline TREWS & Yes & & & No & & & \\
\hline \multicolumn{8}{|l|}{ Age } \\
\hline MEWS & - & - & - & - & - & - & - \\
\hline TREWS & & & & $<40$ & & $41-70$ & $\geq 71$ \\
\hline
\end{tabular}

MEWS modified early warning score, TREWS triage in emergency department early warning score, AVPU A: alert, V: verbal (reacting to voice), P: pain (reacting to pain), U: unresponsive difference in terms of continuous numerical variables where parametric test statistics assumptions were not met was evaluated with the Mann-Whitney $U$ test. One-way ANOVA test was used for comparisons of three and more normally distributed groups, while Kruskal-Wallis test was used in those which did not show normal distribution. Pearson correlation analysis was used to evaluate the relationships between variables showing parametric distribution. Chi-square and Fisher's exact tests were used to analyze whether there was a relationship between categorical variables. ROC curves were drawn to investigate the diagnostic value of MEWS and TREWS in predicting 24-h, 14-day, and 28-day mortalities. $p<0.05$ was considered statistically significant. Results were presented at $95 \%$ confidence interval.

\section{Results}

A total of 339 patients with positive PCR results were included in the study. Of these patients, 54.9\% $(n=186)$ were male and the median age was 65 (IQR 25-75: 25-93). Patients' mean fever was found to be $36.72 \pm 0.64{ }^{\circ} \mathrm{C}$, median SBP value was $122 \mathrm{mmHg}$ (IQR 25-75: 110-135), median heart rate was $80 / \mathrm{min}$ (IQR 25-75: 75-90), and median respiratory rate was $16 / \mathrm{min}$ (IQR 25-75: 12-18).
The median MEWS was calculated as 1 (IQR 25-75: 0-1) whereas the median TREWS was 5 (IQR 25-75: 3-6).

While $35.1 \%$ of the patients $(n=119)$ did not have any comorbidities, $81.4 \%(n=276)$ had typical COVID-19 findings on thorax CT. While $30(8.8 \%)$ of the patients were hospitalized in the intensive care unit, $4(1.2 \%)$ died in the emergency department. Six (1.8\%) of these patients died within the first $24 \mathrm{~h}$. The number of patients who died within 28 days was $57(16.8 \%)$ (Table 2 ).

Mann-Whitney $U$ test was performed to investigate whether there was a difference between MEWS and TREWS with 24-h, 14-day, and 28-day mortality. The results indicated that the MEWS and TREWS of the patients who died within $24 \mathrm{~h}, 14$ days, and 28 days were found to be significantly different $[(p<0.001),(p<0.001)]$. Additionally, the median MEWS was found to be 7 (IQR: 25-75) while the TREWS was 11.5 (IQR 25-75) in mortality within $24 \mathrm{~h}$ (Table 3).

In the ROC analysis conducted to detect the diagnostic value of MEWS and TREWS in predicting 28-day mortality, the area under the curve (AUC) was found to be 0.833 [(95\% CI 0.777-0.888), $(p<0.001)$ ] for MEWS, while the AUC was identified as 0.823 [(95\% CI 0.764-0.882), $(p<0.001))$ ] for TREWS (Fig. 1).

When the cutoff value of the MEWS was 0.5 , the sensitivity was found to be $96.5 \%$ and specificity was $46.8 \%$. When 
Table 2 Clinical and demographic data

\begin{tabular}{|c|c|c|c|}
\hline & & Frequency & Percent \\
\hline \multirow[t]{2}{*}{ Gender } & Female & 153 & 45.1 \\
\hline & Male & 186 & 54.9 \\
\hline \multirow[t]{8}{*}{ Comorbidity } & None & 119 & 35.1 \\
\hline & Hypertension & 24 & 7.1 \\
\hline & Diabetes & 13 & 3.8 \\
\hline & Coronary artery disease & 5 & 1.5 \\
\hline & Asthma/COPD & 18 & 5.3 \\
\hline & Malignancy & 8 & 2.4 \\
\hline & Other & 17 & 5.0 \\
\hline & Coexistence of 2 or more comorbidities & 135 & 39.8 \\
\hline \multirow[t]{4}{*}{ Thorax CT findings } & Typical & 276 & 81.4 \\
\hline & Intermediate & 52 & 15.3 \\
\hline & Atypical & 6 & 1.8 \\
\hline & Negative & 5 & 1.5 \\
\hline \multirow[t]{3}{*}{ Emergency service outcome } & Clinical hospitalization & 305 & 90.0 \\
\hline & Intensive care hospitalization & 30 & 8.8 \\
\hline & Exitus in the emergency & 4 & 1.2 \\
\hline \multirow[t]{2}{*}{ Mortality within $24 \mathrm{~h}$} & No & 333 & 98.2 \\
\hline & Yes & 6 & 1.8 \\
\hline \multirow[t]{2}{*}{ Mortality within 14 days } & No & 291 & 85.8 \\
\hline & Yes & 48 & 14.2 \\
\hline \multirow[t]{2}{*}{ Mortality within 28 days } & No & 282 & 83.2 \\
\hline & Yes & 57 & 16.8 \\
\hline Total & & 339 & 100.0 \\
\hline
\end{tabular}

the cutoff value was 1.5 , the sensitivity was identified as $63.2 \%$ and specificity was $87.2 \%$. When the cutoff value of the TREWS was 4.5 , the sensitivity was found to be $94.7 \%$ and specificity was $52.1 \%$. Finally, when the cutoff value was 5.5 , the sensitivity was identified as $77.2 \%$ and specificity was $67.7 \%$ (Table 4).

\section{Discussion}

With a total of 2.7 million deaths, the COVID-19 pandemic has been continuing to be an international public health emergency worldwide. While mild symptoms are observed in most of the cases, some patients die due to viral pneumonia and multiorgan dysfunction. Quick and accurate identification of serious and critically ill patients enables the correct use of medical resources and effective treatment.

Implementing scoring systems can facilitate effective assessment by emergency or intensive care physicians to screen critically ill patients. However, currently, there is no specific scoring system for evaluating COVID-19 patients. Until a new and effective scoring system is developed, it is appropriate to adopt existing scoring systems to predict the mortality in patients with severe COVID 19 symptoms.
In our study, we evaluated the role of MEWS and TREWS in predicting mortality of COVID-19 patients requiring hospitalization. We found that MEWS and TREWS were significantly higher in the cases of mortality.

Of PCR positive COVID-19 patients with hospitalization indication, $64.9 \%$ had comorbid diseases and often had two or more comorbidities. Similar to our results, comorbidity has been found to be an important determinant in hospitalization and intensive care needs in various studies [18, 19].

Thoracic CT findings for COVID-19 were found in $81.4 \%$ of the patients and $8.8 \%$ had an indication for intensive care hospitalization. Thoracic CT findings are effective in determining the severity of the COVID-19 disease as well as in determining the hospitalization indications and even the need for intensive care [20, 21]. In the studies conducted, intensive care hospitalization rates vary in a range of $21.4-69 \%$ [22, 23]. This rate was found to be low in our study. This may be due to the inclusion of only PCR positive patients in our patient population.

COVID-19 mortality rates vary regionally. In the study conducted by Ciceri et al. with 950 patients in the Italian population, the 30 -day mortality was found to be $17 \%$, while it was reported as $10.7 \%$ in the American population including 8625 patients with positive PCR results [24, 25]. In our study, the 28 -day mortality rate was $16.8 \%$, which is similar to the literature. 


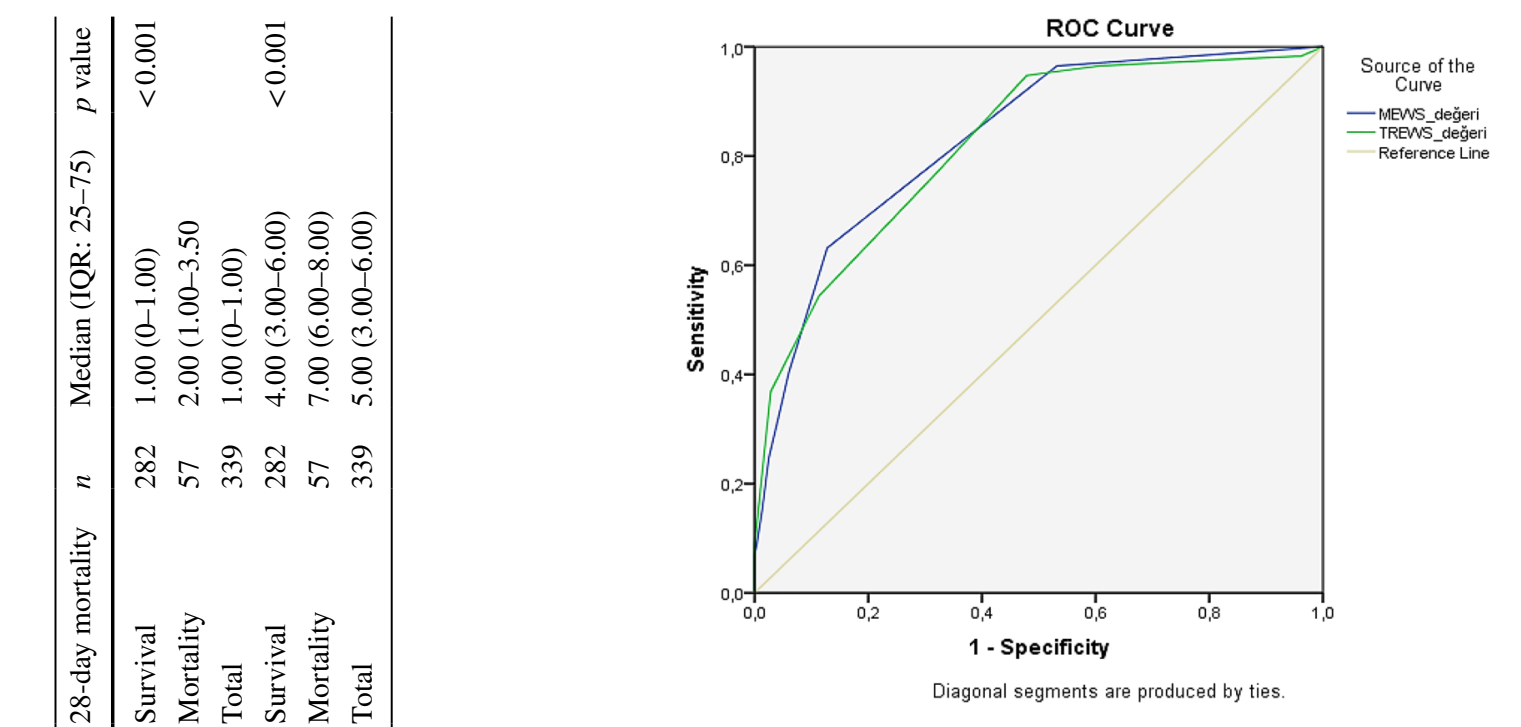

Fig. 1 Area under curve (AUC) values of MEWS and TREWS to predict mortality

When mortality rates were examined, the rate of exitus in the emergency department was found to be $1.2 \%$. Early diagnosis of critical patients and early access to effective treatment modalities may affect mortality rates. At this point, scoring systems can guide emergency physicians. There are many studies conducted to evaluate the efficacy of MEWS which is frequently used in the follow-up of mortality, ICU hospitalization, and prognosis in COVID-19 patients. In the study conducted by Wang et al. with 235 PCR positive COVID-19 patients, the MEWS was found to be effective in predicting mortality; the cutoff value was calculated as 2.5 in males over the age of 75 and 3.5 in the other age group [9].

In order to investigate the use of MEWS in predicting hospital outcomes of emergency patients. A MEWS of higher than 5 within $24 \mathrm{~h}$ following admission was found to have a significant relationship with in-hospital mortality $(p<0.0001)$. The ROC (receiver operator characteristic) curve demonstrated that in patients whose MEWS was $\geq 5$ within $24 \mathrm{~h}$, the AUC was found to be 0.9 . (95\% CI 0.95-0.98). Therefore, it was concluded that MEWS was effective in predicting in-hospital mortality with a sensitivity of $78 \%$ and specificity of $94 \%$. Three hundred patients who were planned to be hospitalized after being evaluated in the emergency department were included in the study. However, the mortality was not evaluated within the first $24 \mathrm{~h}$ in this study [26]. In our study, when the cutoff value was 1.5 for MEWS, the sensitivity was found to be $63.2 \%$ and specificity was $87.2 \%$ (AUC 0.833 $(0.777-0.88895 \% \mathrm{CI}))$. We evaluated that MEWS, which is calculated at the emergency admission, is an important and effective method to identify critically ill patients and to predict 28-day mortality. 
Table 4 ROC analysis according to MEWS and TREWS scores values at diagnosis of 28-day mortality

\begin{tabular}{llllll}
\hline AUC (95\% CI) & $p$ & Risk factor & Cutoff & Sensitivity \% & Specificity \% \\
\hline $0.833(0.777-0.888)$ & $<0.001$ & MEWS & 0.5 & 96.5 & 46.8 \\
& & & 1.5 & 63.2 & 87.2 \\
$0.823(0.764-0.882)$ & $<0.001$ & \multirow{2}{*}{ TREWS } & 3.5 & 96.5 & 94 \\
& & & 4.5 & 94.7 & 39.4 \\
& & & 5.5 & 77.2 & 52.1 \\
& & & & \\
\hline
\end{tabular}

$A U C$ area under curve, $C I$ confidence interval
In another study involving $142 \mathrm{PCR}$ positive patients conducted at the University of Toledo, 14 patients were hospitalized in the intensive care unit and the cutoff value for MEWS was found to be 5 in predicting intensive care admission of these patients (AUC was 0.935) [8]. The significant determinant status of MEWS on mortality may guide physicians in identifying critically ill patients in emergency departments.

In another study conducted by Hu et al. with 319 patients by comparing 5 early warning scores to evaluate critical COVID-19 patients, it was found that NEWS and NEWS2 had a moderate discriminatory power and those scores had a potential as prognostic tools for screening critically ill COVID-19 patients. On the other hand, it was found in a study that MEWS was not a good prognostic predictor. In the study, the AUC for MEWS was found to be 0.670 (95\% CI $0.573-0.767$ ) to predict mortality and MEWS was found not to be a good prognostic predictor for COVID-19 [27]. This significant difference in the results can be explained by the presence of COVID-19 patients in our study population who were only indicated for hospitalization and that these patients were often in the serious and critically ill group.

In our study, we evaluated TREWS as well as MEWS in predicting mortality. Unlike MEWS, we think that TREWS, which includes the patient's age and the need for oxygen support, may be effective in predicting the critical patient due to the current pathology in which hypoxia is at the forefront. In our study, the effective cutoff value of TREWS in predicting 28-day mortality was found to be 5.5 (AUC (95\% CI) 0.823 (0.764-0.882), sensitivity was $77.2 \%$, and specificity was $67.7 \%$ ). Lee et al. found the AUC of the TREWS to be $0.906,0.899,0.853$, and 0.801 for in-hospital mortality within 24 h, 48 h, 7 days, and 30 days, respectively. These results indicate that the prognostic performance of the TREWS to predict in-hospital mortality in emergency patients is superior to those of NEWS and MEWS. In the study, a total of 81,520 patients admitted to the emergency department were retrospectively examined, and all patients over the age of 16 were included but were not associated with COVID-19. Our study supports the effective use of this newly developed scoring system in COVID-19-specific patients [17].
In our study, in $6(1.8 \%)$ cases with 24-h mortality, the median MEWS was found to be 7 (IQR 25-75) while the TREWS was 11.5 (IQR 25-75). In the 28-day mortality group, the median MEWS was identified as 2 (IQR 25-75), while the TREWS value was 7 (IQR 25-75). The calculated scores were found to be significantly higher than those of the surviving group $(p<0.01)$. High TREWSs, which were identified especially in the group with mortality within the first $24 \mathrm{~h}$, can significantly guide emergency physicians in predicting mortality.

\section{Conclusion}

Emergency services are places where fast and efficient patient care and treatment modalities are carried out simultaneously. Especially in situations such as COVID-19 that require effective use of multiple applications and resources, the rapid recognition of the critical patient is vital.

MEWS and TREWS, which are fast and easily calculable scoring methods, can assist physicians in predicting mortality. TREWS can guide the clinical course in the initial evaluation of patients in emergency departments, especially in clinical practice, and can be used effectively to evaluate patient prognosis. We think that this can form the basis for multicenter prospective studies addressing this issue.

Since our study was planned retrospectively, some data regarding patients were lost. We used scoring systems to predict mortality only in severe COVID-19 patients with hospitalization indication. However, we did not evaluate the moderate and mild group followed up according to the disease classification or the patients with negative PCR test and CT findings compatible with COVID-19. We did not include the effects of the treatments applied to hospitalized patients on the 28-day mortality.

Acknowledgements Thanks to Assoc. Prof. Melih Yuksel for his supervision.

Author contribution Huseyin Aygun: design, statistical analysis, and interpretation of data for the work; final approval of the version to 
be published. Suna Eraybar: writing, revising study critically for important intellectual content; final approval of the version to be published.

Availability of data and materials The datasets generated during and/or analyzed during the current study are available from the corresponding author on reasonable request.

\section{Declarations}

Ethical disclosure Ethical committee approval was obtained from Yuksek Ihtisas Education and Research Hospital Ethical committee during the study planning phase. An informed consent form has been signed by the parents of the cases involved.

Competing interests The authors declare no competing interests.

\section{References}

1. Dundar ZD, Ergin M, Karamercan MA et al (2016) Modified early warning score and vitaPac early warning score in geriatric patients admitted to emergency department. Eur J Emerg Med 23(6):406-412

2. Hung SK, Ng CJ, Kuo CF et al (2017) Comparison of the mortality in emergency department sepsis score, modified early warning score, rapid emergency medicine score and rapid acute physiology score for predicting the outcomes of adult splenic abscess patients in the emergency department. PLoS One 12(11): e0187495

3. Subbe CP, Kruger M, Rutherford P et al (2001) Validation of a modified early warning score in medical admissions. QJM 94(10):521-526

4. Bulut M, Cebicci H, Sigirli D et al (2014) The comparison of modified early warning score with rapid emergency medicine score: a prospective multicentre observational cohort study on medical and surgical patients presenting to emergency department. Emerg Med J 31(6):476-481

5. Nannan Panday RS, Minderhoud TC, Alam N et al (2017) Prognostic value of early warning scores in the emergency department (ED) and acute medical unit (AMU): A narrative review. Eur J Intern Med 45:20-31

6. Tirotta D, Gambacorta M, La Regina M et al (2017) Evaluation of the threshold value for the modified early warning score (MEWS) in medical septic patients: a secondary analysis of an Italian multicentric prospective cohort (SNOOPII study). QJM: An Int J Med 110 (6):369-373

7. Churpek MM, Syender A, Han X et al (2017) Quick sepsis releated organ failure assessment, systemic early response syndrome and early warning score for detecting clinical deterioration in infected patients outside the intensive care unit. Am J Respir Crit Care Med 195(7):906-911

8. Wheeler I, Price C, Sitch A et al (2013) Early warning scores generated in development healthcare settings are not sufficient at predicting early mortality in Blantye, Malawi: A prospective Cohort study. PLoS One 8(3):e59830

9. Bhatnagar M, Sirohi N, Dubey AB (2021) Prediction of hospital outcome in emergency medical admissions using modified early warning score (MEWS): Indian experience. J Family Med Prim Care 10(1)

10. Aygün H, Eraybar S, Özdemir F et al (2020) Predictive value of modified early warning scoring system for identifying critical patients with malignancy in emergency department. Arch Iran Med 23(8):536-541
11. Reini K, Fredrikson M, Oscarsson A (2012) The prognostic value of the modified early warning score in critically ill patients.o prospective, observational study. Eur J Anesthesiol 29(3):152-7

12. Barnett WR, Radhakrishnan M, Macko J et al (2021) Initial MEWS score to predict ICU admission or transfer of hospitalized patients with COVID-19: a retrospective study. Journal of Infectin 82(2):282-327

13. Covino M, Sandromi C, Santoro M et al (2020) Predicting intensive care unit admission and deathfor COVID-19 patients in the emergency department using early warning scores. Resuscitation 156 :84-91

14. Wang L, Lv Q, Zhang X et al (2020) The utility of MEWS for predicting the mortality in the elderly adults with COVID_19: a retrospective cohort study with comparison to other predictive clinical scores. PeerJ 28:e10018

15. Ciceri F, Castagna A, Rovere-Querini P et al (2020) Early predictors of clinical outcomes of Covid-19 outbreak in Milan, İtaly. Clin Immunol 217:108509

16. Hu H, Yao N (2020) Qiu Y The comparison of REMS and MEWS for COVID-19 patients less than 65 years of Age. Acad Emerg Med $11: 1215$

17. Lee SB, Kim DH, Kim T et al (2020) Emergency Department Triage Early Warning Score (TREWS) predicts in-hospital mortality in the emergency department. Am J Emerg Med 38(2):203-210

18. Chen N, Zhou M, Dong X et al (2020) Epidemiological and clinical characteristics of 99 cases of 2019 novel coronavirus pneumonia in Wuhan, China: a descriptive study. Lancet 395(10223):507-513

19. Zhu YC, Tan L, Liu L et al (2020) Comparative analysis of characteristics and medications between corona virus disease 2019 and several acute reproductive syndrome. Clin Med J 18:15-23

20. Ai T, Yang Z, Hou H et al (2020) Correlation of chest CT and RTPCR testing in coronavirus disease 2019 (COVID-19) in China: a report of 1014 cases. Radiology 296(2):E32-E40

21. National Health Commission of the People's Republic of China (2020) Novel coronavirus pneumonia diagnosis and treatment program (5th trial version) (in Chinese) http://www.nhc.gov. cn/xcs/zhengcwj/202002/d4b895337e19445f8d728fcaf1e3e13a. shtml. Accessed 2 Mar 2020

22. Chang R, Elhusseiny KM, Yeh YC et al (2021) COVID-19 ICU and mechanical ventilation patient caracteristics and outcomes-a systemic review and meta-analysis. PLoS One 16 (2):e0246318

23. Ranzani OT, Bastos LS, Gelli JG et al (2021) Characterisation of the first 250000 hospital admissions for Covid-19 in Brazil: a retrospective analysis of nationwide data. Lancet Respir Med 9:407-418

24. Ciceri F, Ruggeri A, Lembo R et al (2020) Decreased in hospital mortality in patients with Covid-19 pneumonia. Pathog Glob Health 114(6):281-282

25. Rentsch CT, Kidwai-Khan F, Tate JP et al (2020) Patterns of Covid19 testing and mortality by race and ethnicity among United States veterans: a nationwide cohort study. PLoS Med 17(9):e1003379

26. Bhatnagar M, Sirohi N, Dubey AB (2021) Prediction of hospital outcome in emergency medical admissions using modified early warning score (MEWS): Indian experience. J Family Med Prim Care 10:192-198

27. $\mathrm{Hu}$ H, Yao N, Qlu Y (2020) Predictive value of 5 early warning scores for critical COVID-19 patients. Disaster Med Public Health Prep 9:1-8

Publisher's Note Springer Nature remains neutral with regard to jurisdictional claims in published maps and institutional affiliations. 